\title{
ON THE TWO-DIMENSIONAL COMPRESSIBLE ISENTROPIC NAVIER-STOKES EQUATIONS
}

\author{
Catherine Giacomoni $^{1}$ and Pierre Orenga ${ }^{1}$
}

\begin{abstract}
We analyze the compressible isentropic Navier-Stokes equations (Lions, 1998) in the twodimensional case with $\gamma=c_{p} / c_{v}=2$. These equations also modelize the shallow water problem in height-flow rate formulation used to solve the flow in lakes and perfectly well-mixed sea. We establish a convergence result for the time-discretized problem when the momentum equation and the continuity equation are solved with the Galerkin method, without adding a penalization term in the continuity equation as it is made in Lions (1998). The second part is devoted to the numerical analysis and mainly deals with problems of geophysical fluids. We compare the simulations obtained with this compressible isentropic Navier-Stokes model and those obtained with a shallow water model (Di Martino et al., 1999). At first, the computations are executed on a simplified domain in order to validate the method by comparison with existing numerical results and then on a real domain: the dam of Calacuccia (France). At last, we numerically implement an analytical example presented by Weigant (1995) which shows that even if the data are rather smooth, we cannot have bounds on $\rho$ in $L^{p}$ for $p$ large if $\gamma<2$ when $N=2$.
\end{abstract}

Mathematics Subject Classification. 35Q30.

Received: September 7, 2001. Revised June 26, 2002.

\section{INTRODUCTION}

This paper is devoted to the analysis of the two-dimensional compressible isentropic Navier-Stokes equations for which existence results have been established by Lions [6]. We introduce another construction of approximate solutions of the problem by using the Galerkin method which induces a simpler numerical approximation. Let $\Omega$ be a bounded simply connected (to simplify) smooth open domain in $\mathbb{R}^{2}$ with boundary $\Gamma$ and let $Q$ be the cylinder $Q=\Omega \times] 0, T[$ with its boundary $\Sigma=\Gamma \times] 0, T[$.

We consider the following Cauchy problem

$$
\begin{aligned}
& \frac{\partial \rho}{\partial t}+\operatorname{div}(\rho u)=0 \text { in } Q, \\
& \frac{\partial \rho u_{i}}{\partial t}+\operatorname{div}\left(\rho u u_{i}\right)-\mu \Delta u_{i}+\partial_{i}\left(a \rho^{\gamma}\right)=\rho f_{i}, 1 \leq i \leq 2, \text { in } Q,
\end{aligned}
$$

Keywords and phrases. Navier-Stokes, compressible, shallow water, time-discretisation, Galerkin.

1 Systèmes Physiques de l'Environnement, UMR CNRS 6134, Université de Corse, Quartier Grossetti, BP 52, 20250 Corte, France. e-mail: giaco@univ-corse.fr, orenga@univ-corse.fr 
where $\rho \geq 0$ corresponds to the fluid density and $u$ a vector-valued (in $\mathbb{R}^{2}$ ) function that corresponds to its velocity. The function $f=f(x, t)$ is a given function corresponding to the force terms on $Q, \mu$ is a positive viscous coefficient and we consider the case $\gamma=2$ in order to compare this model with the shallow water model in the numerical part. These equations modelize the shallow water problem in height-flow rate formulation.

We specify initial conditions

$$
\rho(t=0)=\rho_{0}(x) \in \Omega, \quad \rho u(t=0)=m_{0}(x) \in \Omega
$$

and we assume that $\rho_{0}$ and $m_{0}$ satisfy

$$
\begin{aligned}
& \rho_{0} \in L^{\gamma}(\Omega), \rho_{0} \geq 0 \text { a.e. in } \Omega, \rho_{0} \neq 0, \\
& m_{0} \in L^{\frac{2 \gamma}{\gamma+1}}(\Omega), m_{0}=0 \text { a.e. on }\left\{\rho_{0}=0\right\}, \\
& \frac{\left|m_{0}\right|^{2}}{\rho_{0}} \in L^{1}(\Omega), \quad\left(\frac{\left|m_{0}\right|^{2}}{\rho_{0}}=0 \text { on }\left\{\rho_{0}=0\right\}\right) .
\end{aligned}
$$

We must add boundary conditions to the system $(1,2)$. We use classical boundary conditions to geophysical fluids and particularly, for the shallow water models

$$
u \cdot \mathrm{n}=0 \text { and } \operatorname{curl} u=0 \text { on } \Sigma .
$$

The first condition is a natural condition of impermeability type on the normal velocity where $\mathrm{n}$ denotes the unit outward normal to $\Gamma$. The second condition can be interpreted as a viscous term dissipation at the boundary (more exactly a non-dissipation since this term is equal to zero) [8]. These conditions are more suitable to geophysical fluid equations than a classical Dirichlet condition which generates very expensive calculations of boundary layer. Moreover, these conditions allows to solve the problem with the Galerkin method using a special basis which permits to write the Hodge-Helmoltz decomposition of vector fields as sum of gradient and Curl of scalar fields [4]. It is this decomposition which allows to obtain the necessary compactness to pass to the limit in the equations.

Notice that the resolution of these equations can be extended to the case of periodic boundary conditions for instance, and for values of $\gamma$ different from 2 using the notion of renormalized solutions of the continuity equation [2]. Indeed, in this case, the Galerkin method is not directly valid because the energy estimates are not immediately obtained. In order to avoid this difficulty, we can solve the system $(1,2)$ by making the change of variable $\beta(\rho)=\rho^{\gamma / 2}$. The continuity equation is then solved under the following form

$$
\frac{\partial \rho^{\gamma / 2}}{\partial t}+\operatorname{div}\left(\rho^{\gamma / 2} u\right)+\left(\frac{\gamma}{2}-1\right)(\operatorname{div} u) \rho^{\gamma / 2}=0
$$

and we verify easily that we obtain the estimates.

We use a time-discretization method to solve this problem and the main differences with the existence proof developed in [6] are that we use the Galerkin method to solve the time-discretized problem and we do not need to penalize the continuity equation. In particular, we show that we have enough compactness on the Galerkin solutions to pass to the limit in the equations. Let us note that this compactness is obtained thanks to the boundary conditions used and in the case of Dirichlet boundary conditions, we do not manage to obtain it.

In the numerical part, we solve this problem that we compare with a shallow water model in which the diffusion term depends on $\rho$. At last, we consider an analytic case introduced by Weigant [11] that shows the formation of singularities in finite time for smooth solutions of $(1,2)$.

The numerical results show that the proposed numerical method is well adapted to this kind of problems. 
For the following analysis, we define the functional space $\mathcal{V}$ by

$$
\begin{aligned}
\mathcal{V} & =\left\{\varphi \in L^{2}(\Omega)^{2} / \operatorname{div} \varphi \in L^{2}(\Omega), \operatorname{curl} \varphi \in L^{2}(\Omega), \varphi \cdot \mathrm{n}=0 \text { on } \gamma\right\} \\
& =\left\{\varphi \in H^{1}(\Omega)^{2}, \varphi \cdot \mathrm{n}=0 \text { on } \gamma\right\}
\end{aligned}
$$

equipped with the graph-norm

$$
\|\varphi\|_{\mathcal{V}}^{2}=\|\varphi\|_{L^{2}(\Omega)^{2}}^{2}+\|\operatorname{div} \varphi\|_{L^{2}(\Omega)}^{2}+\|\operatorname{curl} \varphi\|_{L^{2}(\Omega)}^{2}
$$

Notice that $\mathcal{V}$ corresponds to the space $H_{0}(\operatorname{div} ; \Omega) \cap H(\operatorname{curl} ; \Omega)[4]$ and $v \in \mathcal{V}$ can be split as follows:

$$
v=\nabla p+\operatorname{Curl} q
$$

where $\frac{\partial p}{\partial \mathrm{n}}=0$ and $q=0$ on $\gamma$. We denote by $A(u, \varphi)$ the bilinear form $A(u, \varphi)=(\operatorname{div} u, \operatorname{div} \varphi)+(\operatorname{curl} u, \operatorname{curl} \varphi)$ where $(\cdot, \cdot)$ represents the scalar product in $L^{2}(\Omega)$ or $L^{2}(\Omega)^{2}$. Notice that when $\Omega$ is simply connected then $A(u, u)$ is an equivalent norm on $\mathcal{V}$. However, if $\Omega$ is not simply connected, one can prove [8] that we can obtain a bound on $u$ in $\mathcal{V}$.

We present the special basis of $\mathcal{V}$ used afterwards [9]

$$
\text { (B) }\left\{\begin{aligned}
-\Delta u & =\lambda u & & \text { in } \Omega \\
u \cdot \mathrm{n} & =0 & & \text { on } \Gamma \\
\operatorname{curl} u & =0 & & \text { on } \Gamma,
\end{aligned}\right.
$$

for which the solutions can be obtained by solving the following scalar problems (when $\Omega$ is simply connected)

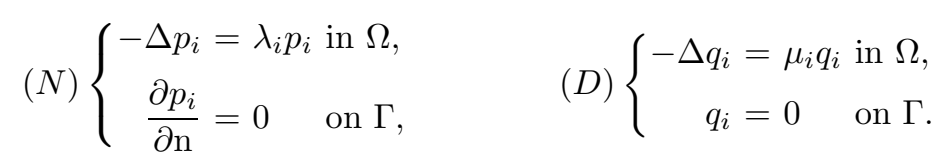

The set of $\left\{\nabla p_{i}, i \in \mathbb{N}^{*}\right\} \cup\left\{\operatorname{Curl} q_{i}, i \in \mathbb{N}^{*}\right\}$ is an orthogonal basis of $L^{2}(\Omega)^{2}$ and $\mathcal{V}$, and is dense in $L^{p}(\Omega)^{2}$, $p<\infty$. Notice that if $\Gamma$ is of class $C^{m-1,1}$, then $\nabla p_{i}$ and $\operatorname{Curl} q_{i} \in H^{m}(\Omega)^{2}$.

Let us recall the following theorem which is the main existence result established in [6]. Assuming the conditions (3) and $f \in L^{1}\left(0, T ; L^{\frac{2 \gamma}{\gamma-1}}(\Omega)^{2}\right)$, the solutions $(\rho, u)$ satisfying $(1,2)$ in the sense of distributions are such that

$$
\begin{aligned}
& \rho \geq 0, \rho \in L^{\infty}\left(0, T ; L^{\gamma}(\Omega)\right) \cap C\left([0, T] ; L^{q}(\Omega)\right), 1 \leq q<\gamma, \\
& u \in L^{2}(0, T ; \mathcal{V}), \\
& \rho|u|^{2} \in L^{\infty}\left(0, T ; L^{1}(\Omega)\right), \\
& \rho u \in C\left([0, T] ; L^{\frac{2 \gamma}{\gamma+1}}(\Omega)^{2}-\omega\right) .
\end{aligned}
$$

Theorem 0.1. Under the above conditions, there exists a solution $(\rho, u)$ of $(1,2)$ satisfying the initial conditions (3) and such that $\rho \in L^{p}(\Omega \times(0, T))$ where $p=\gamma+\frac{2}{N} \gamma-1$. In addition, $(\rho, u)$ satisfies the following energy inequality for almost all $t \in[0, T]$

$$
\int_{\Omega} \frac{1}{2} \rho|u|^{2}+\frac{a}{\gamma-1} \rho^{\gamma} \mathrm{d} x+\mu A(u, u) \leq \int_{\Omega} \frac{1}{2} \frac{\left|m_{0}\right|^{2}}{\rho_{0}}+\frac{a}{\gamma-1} \rho_{0}^{\gamma} \mathrm{d} x+\int_{0}^{t} \mathrm{~d} s \int_{\Omega} \mathrm{d} x \rho f \cdot u .
$$

One can see that, with these estimates, the passage to the limit in the pressure term $\rho^{\gamma}$ is the main difficulty. 


\section{Time-Discretization}

In [6], a first step to solve the equations $(1,2)$ is based on a classical Euler time-discretization. In this section, we prove the existence of solutions of this time-discretized problem by using the Galerkin method. So, in this work we analyze the following stationary problem

$$
\begin{aligned}
& \alpha \rho+\operatorname{div}(\rho u)=h \text { in } \Omega \\
& \alpha \rho u+\operatorname{div}(\rho u \otimes u)-\mu \Delta u+a \nabla\left(\rho^{2}\right)=\rho f+g \text { in } \Omega
\end{aligned}
$$

where $\alpha=\frac{1}{\Delta t}>0$ and $f, g, h$ are given functions defined by $h=\frac{1}{\Delta t} \rho(t-\Delta t), h \geq 0$ in $\Omega, h \not \equiv 0$ and $g=\frac{1}{\Delta t} \rho(t-\Delta t) u(t-\Delta t)$.

Always in [6], in order to establish the existence of solutions for this problem, the author approximates (10, 11) by the following penalized problem

$$
\begin{aligned}
& \alpha \rho+\operatorname{div}(\rho u)-\epsilon \Delta \rho=h \\
& \frac{h u}{2}+\frac{1}{2} \rho u \cdot \nabla u+\alpha \frac{\rho u}{2}+\frac{1}{2} \operatorname{div}(\rho u \otimes u)-\mu \Delta u+a \nabla\left(\rho^{2}\right)=\rho f+g
\end{aligned}
$$

where $\epsilon>0$. The existence of solutions to this problem is shown by a Leray-Schauder fixed point method. Notice that one adds to $(12,13)$ the Neumann boundary condition (for instance) on $\rho, \frac{\partial \rho}{\partial n}=0$ on $\Gamma$.

In this paper, in order to approximate the solution of discretized problem $(10,11)$, we use the Galerkin method for the momentum and continuity equations which allows to circumvent the use of the penalization in the continuity equation thanks to the regularity of the special basis. We apply the Leray-Schauder theorem in order to construct the approximate solutions and moreover, we show that we have enough compactness on $\rho$ to pass to the limit.

The momentum equation can be written in the following way

$$
u(\alpha \rho+\operatorname{div}(\rho u))+\rho u \cdot \nabla u-\mu \Delta u+a \nabla\left(\rho^{2}\right)=\rho f+g \text { in } \Omega
$$

and since, we have

$$
\alpha \rho+\operatorname{div}(\rho u)=h \text { in } \Omega,
$$

it is equivalent to solve the following problem

$$
\begin{aligned}
& \alpha \rho+\operatorname{div}(\rho u)=h \text { in } \Omega, \\
& h u+\rho u \cdot \nabla u-\mu \Delta u+a \nabla\left(\rho^{2}\right)=\rho f+g \text { in } \Omega .
\end{aligned}
$$

This problem is solved under the following weak formulation $(W)$

$$
\begin{aligned}
& \alpha(\rho, \psi)+(\operatorname{div}(\rho u), \psi)=(h, \psi), \forall \psi \in H^{1}(\Omega), \\
& (h u, \varphi)+(\rho u \cdot \nabla u, \varphi)+\mu A(u, \varphi)+a\left(\nabla\left(\rho^{2}\right), \varphi\right)=(\rho f, \varphi)+(g, \varphi), \forall \varphi \in \mathcal{V} \cap H^{4}(\Omega)^{2} .
\end{aligned}
$$

We consider $\mathcal{V}_{n}$ the space generated by the $n$ first elements of the basis $(B)$ of $\mathcal{V}$ and $H^{1}(\Omega)_{m}$ represents the subspace of $H^{1}(\Omega)$ generated by the functions $\left\{p_{1}, \ldots, p_{m}\right\}$, solutions of problem $(N)$. So, $u_{n, m}$ and $\rho_{n, m}$ are under the form

$$
u_{n, m}=\sum_{i=1}^{n} a_{i}(t) \varphi_{i}(x), \quad \rho_{n, m}=\sum_{i=1}^{m} b_{i}(t) p_{i}(x)
$$


We approach $(W)$ by the following problem $\left(W_{n, m}\right), n$ fixed

$$
\begin{aligned}
& \alpha\left(\rho_{n, m}, p_{i}\right)+\left(\operatorname{div}\left(\rho_{n, m} u_{n, m}\right), p_{i}\right)=\left(h_{n, m}, p_{i}\right), \forall p_{i} \in H^{1}(\Omega)_{m} \\
& \left(h_{n, m} u_{n, m}, \varphi_{i}\right)+\left(\rho_{n, m} u_{n, m} \cdot \nabla u_{n, m}, \varphi_{i}\right)+\mu A\left(u_{n, m}, \varphi_{i}\right)+a\left(\nabla\left(\rho_{n, m}\right)^{2}, \varphi_{i}\right)=\left(\rho_{n, m} f, \varphi_{i}\right) \\
& +\left(g_{n, m}, \varphi_{i}\right), \forall \varphi_{i} \in \mathcal{V}_{n}
\end{aligned}
$$

and we note $\left(W_{n}\right)$ the problem after the passage to the limit on $m$ :

$$
\begin{aligned}
& \alpha\left(\rho_{n}, p_{i}\right)+\left(\operatorname{div}\left(\rho_{n} u_{n}\right), p_{i}\right)=\left(h_{n}, p_{i}\right), \forall p_{i} \in H^{1}(\Omega) \\
& \left(h_{n} u_{n}, \varphi_{i}\right)+\left(\rho_{n} u_{n} \cdot \nabla u_{n}, \varphi_{i}\right)+\mu A\left(u_{n}, \varphi_{i}\right)+a\left(\nabla\left(\rho_{n}\right)^{2}, \varphi_{i}\right)=\left(\rho_{n} f, \varphi_{i}\right)+\left(g_{n}, \varphi_{i}\right), \forall \varphi_{i} \in \mathcal{V}_{n} .
\end{aligned}
$$

Notice that $W_{n}$ verifies the continuity equation exactly, that is necessary for the passage to the limit in $n$.

We assume that the data verify

$$
h \geq 0, h \not \equiv 0, h \in L^{2}(\Omega), g \in L^{p}(\Omega)^{2}, p<2 \text { and } f \in L^{4}(\Omega)^{2} .
$$

Replacing $\varphi$ by $u$ in equation (19) and using the relation $u \cdot \nabla\left(\rho^{2}\right)=2\left(\alpha \rho^{2}-h \rho+\operatorname{div}\left(\rho^{2} u\right)\right)$, we obtain

$$
\int_{\Omega} h \frac{|u|^{2}}{2}+\alpha \rho \frac{|u|^{2}}{2}+2 a\left(\alpha \rho^{2}-h \rho\right)+\mu A(u, u) \mathrm{d} x=\int_{\Omega} \rho u \cdot f+u \cdot g \mathrm{~d} x .
$$

We can now state the following theorem:

Theorem 1.1. Assuming (24), the sequence of solutions $\left(\rho_{n, m}, u_{n, m}\right)$ of $\left(W_{n, m}\right)$ converges uniformly in $n$ to $\left(\rho_{n}, u_{n}\right)$ solution of problem $\left(W_{n}\right)$ with $\left(\rho_{n}, u_{n}\right)$ satisfying

$$
\begin{aligned}
& \rho_{n} \text { is bounded in } L^{4}(\Omega), \\
& \nabla\left(\operatorname{div} u_{n}-\frac{a}{\mu} \rho_{n}^{2}\right) \text { is bounded in } L^{r}(\Omega)^{2}, r<\frac{4}{3},
\end{aligned}
$$

and $\left(\rho_{n}, u_{n}\right)$ converges to $(\rho, u)$ which verifies the energy equation (25).

The proof of this theorem is built up on these following steps

- Construction of approximate solutions of $(18,19)$ (Sect. 1.1).

- Bound on $\rho_{n}$ in $L^{4}(\Omega)$ (Sect. 1.2).

- Bound on $\operatorname{div} u_{n}-b \rho_{n}^{2}$ in $W^{1, r}(\Omega), r<\frac{4}{3}$, where $b=\frac{a}{\mu}$ (Sect. 1.3).

- Compactness result on $\rho_{n}$ (Sect. 1.4).

\subsection{Construction of approximate solutions}

We establish a first result:

Lemma 1.2. $\left(W_{n}\right)$ has a solution $\left(\rho_{n}, u_{n}\right)$ satisfying

$$
\left(\rho_{n}, u_{n}\right) \in H^{1}(\Omega) \times H^{4}(\Omega)^{2} .
$$

Proof. We consider the following problem where $v$ is fixed, $v \in H^{3}(\Omega)^{2}$ :

$$
\begin{aligned}
& \alpha\left(\rho_{n, m}, p_{i}\right)+\left(\operatorname{div}\left(\rho_{n, m} u_{n, m}\right), p_{i}\right)=\left(h_{n, m}, p_{i}\right), \forall p_{i} \in H^{1}(\Omega)_{m} \\
& \left(h_{n, m} u_{n, m}, \varphi_{i}\right)+\left(\rho_{n, m} v \cdot \nabla v, \varphi_{i}\right)+\mu A\left(u_{n, m}, \varphi_{i}\right)+a\left(\nabla\left(\rho_{n, m}\right)^{2}, \varphi_{i}\right)=\left(\rho_{n, m} f, \varphi_{i}\right)+\left(g_{n, m}, \varphi_{i}\right), \forall \varphi_{i} \in \mathcal{V}_{n}
\end{aligned}
$$


Let us write the estimates on $\rho_{n, m}$ and $u_{n, m}$. On the one hand, replacing $p_{i}$ by $\rho_{n, m}$ in (29), we obtain:

$$
\alpha\left\|\rho_{n, m}\right\|_{L^{2}(\Omega)}^{2}+\frac{1}{2} \int_{\Omega} \operatorname{div} u_{n, m} \rho_{n, m}^{2} \mathrm{~d} x=\int_{\Omega} h_{n, m} \rho_{n, m} \mathrm{~d} x
$$

On the other hand, replacing $\varphi_{i}$ by $u_{n, m}$ in (30), we obtain

$$
\begin{aligned}
& \int_{\Omega} h_{n, m} u_{n, m}^{2} \mathrm{~d} x+\int_{\Omega} \rho_{n, m} u_{n, m} v \cdot \nabla v \mathrm{~d} x+\mu A\left(u_{n, m}, u_{n, m}\right)-a \int_{\Omega} \rho_{n, m}^{2} \operatorname{div} u_{n, m} \mathrm{~d} x=\int_{\Omega} \rho_{n, m} u_{n, m} \cdot f \mathrm{~d} x \\
& +\int_{\Omega} g_{n, m} \cdot u_{n, m} \mathrm{~d} x
\end{aligned}
$$

Multiplying (31) by $2 a$ and summing with (32), we get

$$
\begin{array}{r}
2 a \alpha\left\|\rho_{n, m}\right\|_{L^{2}(\Omega)}^{2}+\int_{\Omega} h_{n, m} u_{n, m}^{2} \mathrm{~d} x+\int_{\Omega} \rho_{n, m} u_{n, m} v \cdot \nabla v \mathrm{~d} x+\mu\left\|u_{n, m}\right\|_{\mathcal{V}_{n}}^{2}=2 a \int_{\Omega} h_{n, m} \rho_{n, m} \mathrm{~d} x+\int_{\Omega} \rho_{n, m} u_{n, m} \cdot f \mathrm{~d} x \\
+\int_{\Omega} g_{n, m} \cdot u_{n, m} \mathrm{~d} x . \quad(33)
\end{array}
$$

Thus, we obtain bounds on $u_{n, m}$ in $\mathcal{V}_{n}$ and on $\rho_{n, m}$ in $L^{2}(\Omega)$. We can extract two sequences still noted $u_{n, m}$ and $\rho_{n, m}$ which converge weakly respectively to $u_{n}^{*}, \rho_{n}^{*}$, when $m$ goes to $+\infty$.

The following step consists in the passage to the limit when $m$ goes to $+\infty$. The main difficulty is due to the term $\nabla \rho_{n, m}^{2}$. The bound on $\rho_{n, m}$ in $L^{2}(\Omega)$ is not sufficient to pass to the limit in this term. We must show that $\rho_{n, m}$ is bounded in $H^{1}(\Omega)$ in order to obtain a strong convergence on $\rho_{n, m}$. Then we can pass to the limit in each term of $(29,30)$.

Taking the gradient of the continuity equation, multiplying by $\nabla \rho_{n, m}$ and integrating over $\Omega$, we obtain

$$
\left\|\nabla \rho_{n, m}\right\|_{L^{2}(\Omega)^{2}}^{2}\left(\alpha-\epsilon-C\left\|D u_{n, m}\right\|_{L^{\infty}(\Omega)}\right) \leq C_{\epsilon}\left\|\rho_{n, m}\right\|_{L^{2}(\Omega)}\left\|\nabla \operatorname{div} u_{n, m}\right\|_{L^{2}(\Omega)^{2}}^{2}
$$

Choosing $\Delta t$ such that $\alpha-\epsilon-C\left\|D u_{n, m}\right\|_{L^{\infty}(\Omega)}>0$ where $C$ is a positive constant, we get the bound on $\rho_{n, m}$ in $H^{1}(\Omega)$ thanks to the regularity of the functions basis. Indeed, $u_{n, m}$ is bounded in $H^{4}(\Omega)^{2}$ then $\nabla \operatorname{div} u_{n, m}$ is bounded in $L^{\infty}(\Omega)$.

Finally, we prove the existence of solutions to the problem $\left(W_{n}\right)$ with the Leray-Schauder fixed point theorem [12]. For $t \in[0,1]$ and for all $(\phi, v) \in L^{2}(\Omega) \times H^{3}(\Omega)^{2}$, we define $A_{t}(\phi, v)=\left(\rho_{n}, u_{n}\right)$ as the solution of the following problem $\left(W_{n}\right)^{t}$

$$
\begin{aligned}
& \alpha\left(\rho_{n}, p_{i}\right)+\left(\operatorname{div}\left(\rho_{n} v\right), p_{i}\right)=\left(t h_{n}, p_{i}\right), \rho_{n} \in H^{1}(\Omega) \\
& \left(h_{n} u_{n}, \varphi_{i}\right)+\left(\rho_{n} v \cdot \nabla v, \varphi_{i}\right)+\mu A\left(u_{n}, \varphi_{i}\right)+a\left(\nabla\left(\rho_{n}^{2}\right), \varphi_{i}\right)=\left(\rho_{n}(t f), \varphi_{i}\right)+\left(g_{n}, \varphi_{i}\right), u_{n} \in H^{4}(\Omega)^{2}
\end{aligned}
$$

It is easy to check that $A_{t}$ is a compact mapping on $L^{2}(\Omega) \times H^{3}(\Omega)^{2}$ that depends continuously on parameter $t \in[0,1]$ and that $A_{0}(\phi, v)=(0,0)$. Moreover, each fixed point $\left(\rho_{n}, u_{n}\right)$ of $A_{t}$ solves

$$
\begin{aligned}
& \alpha\left(\rho_{n}, p_{i}\right)+\left(\operatorname{div}\left(\rho_{n} u_{n}\right), p_{i}\right)=\left(t h_{n}, p_{i}\right), \forall p_{i} \in H^{1}(\Omega) \\
& \left(h_{n} u_{n}, \varphi_{i}\right)+\left(\rho_{n} u_{n} \cdot \nabla u_{n}, \varphi_{i}\right)+\mu A\left(u_{n}, \varphi_{i}\right)+a\left(\nabla\left(\rho_{n}^{2}\right), \varphi_{i}\right)=\left(\rho_{n}(t f), \varphi_{i}\right)+\left(t g_{n}, \varphi_{i}\right), \forall \varphi_{i} \in \mathcal{V}_{n} \cap H^{4}(\Omega)^{2}
\end{aligned}
$$

where $\left(\rho_{n}, u_{n}\right)$ is bounded in $H^{1}(\Omega) \times H^{4}(\Omega)^{2}$ uniformly in $t \in[0,1]$. Applying the Leray-Schauder principle, we deduce the existence of a solution of the previous problem for $t=1$, i.e. for $(22,23)$. 


\subsection{Bounds on $\rho_{n}$ in $L^{4}(\Omega)$}

We prove in this section a regularity result which shows that $\rho_{n}$ solution of $(22)$ is bounded in $L^{4}(\Omega)$. Replacing $\varphi$ by $\nabla p_{i}$ in (23), using the special basis defined in the previous section and multiplying by $\frac{-1}{\lambda_{i}}$, we have

$$
a \int_{\Omega} \rho_{n}^{2} p_{i} \mathrm{~d} x=\int_{\Omega}\left(-h_{n} u_{n}-\rho_{n} u_{n} \cdot \nabla u_{n}+\rho_{n} f+g_{n}\right) \frac{\nabla p_{i}}{\lambda_{i}} \mathrm{~d} x+\mu \int_{\Omega} \operatorname{div} u_{n} p_{i} \mathrm{~d} x .
$$

We first prove that $\rho_{n}$ is bounded in $L^{3}(\Omega)$. Multiplying $(35)$ by $\left[P_{n}\left(\rho_{n}^{2}\right)\right]^{1 / 2} p_{i}$ where $P_{n}$ is the projection operator on $\left\{p_{1}, \ldots, p_{n}\right\}$ and summing on $i$, we obtain

$$
\left[P_{n}\left(\rho_{n}^{2}\right)\right]^{3 / 2}=\frac{1}{a}\left[P_{n}\left(\rho_{n}^{2}\right)\right]^{1 / 2} \sum_{i}\left[\int_{\Omega}\left(-h_{n} u_{n}-\rho_{n} u_{n} \cdot \nabla u_{n}+\rho_{n} f+g_{n}\right) \frac{\nabla p_{i}}{\lambda_{i}} \mathrm{~d} x+\mu \int_{\Omega} \operatorname{div} u_{n} p_{i} \mathrm{~d} x\right] p_{i}
$$

In this equation, we just need to bound the term $\rho_{n} u_{n} \cdot \nabla u_{n}$. We adapt the strategy of Lions in [6] which consists in decomposing $\rho_{n} u_{n}=\nabla \theta_{n}+\operatorname{Curl} q_{n}$. Since $\rho_{n} u_{n} \in L^{r}(\Omega)^{2}, \frac{1}{r}=\frac{1}{2}+\frac{1}{q}, q<\infty$, there exists $\nabla \theta_{n}$ and $\operatorname{Curl} q_{n} \in L^{r_{1}}(\Omega)^{2}, 1<r_{1} \leq r<2$ such that

$$
\rho_{n}\left(u_{n} \cdot \nabla\right) u_{n}=\left(\nabla \theta_{n} \cdot \nabla\right) u_{n}+\left(\operatorname{Curl} q_{n} \cdot \nabla\right) u_{n}
$$

Since $\operatorname{div} \nabla \theta_{n}=\operatorname{div}\left(\rho_{n} u_{n}\right)=h_{n}-\alpha \rho_{n}$ is bounded in $L^{2}(\Omega)$ and in addition $\theta_{n} \in W^{1,4 / 3}(\Omega)$, then $\nabla \theta_{n}$ is bounded in $H^{1}(\Omega)^{2} \hookrightarrow L^{\beta}(\Omega)^{2}, \beta<\infty$. So $\left(\nabla \theta_{n} \cdot \nabla\right) u_{n}$ is bounded in $L^{1}(\Omega)^{2}$ (at least) and there exists $\nabla \theta_{n, 1}$ and $\operatorname{Curl} q_{n, 1}$ such that

$$
\left(\nabla \theta_{n} \cdot \nabla\right) u_{n}=\nabla \theta_{n, 1}+\operatorname{Curl} q_{n, 1}
$$

with $\theta_{n, 1}$ bounded in $L^{2}(\Omega)$ and $q_{n, 1} \in H_{0}^{1}(\Omega)$.

Next, since $\operatorname{div} \operatorname{Curl} q_{n}=\operatorname{curl}\left(\nabla u_{n}^{j}\right)=0,1 \leq j \leq N$, we can apply the results of Coifman et al. [1] and deduce that the product of $\operatorname{Curl} q_{n}$ by $\nabla u_{n}^{j}$ belongs to the Hardy space $\mathcal{H}^{q}(\Omega)^{2}$ where $1<\frac{1}{q}=\frac{1}{r}+\frac{1}{2}<1+\frac{1}{N}$, $\frac{2}{3}<q<1$. Since Sobolev's embeddings are also valid in $\mathcal{H}^{q}(\Omega)^{2}$ (equals to $L^{q}(\Omega)^{2}$ if $q>1$ ) with $\frac{N}{N+1}<q<1$, then $\mathcal{H}^{q}(\Omega)^{2} \hookrightarrow W^{-1, q^{*}}(\Omega)^{2}$ where $\frac{1}{q^{*}}=\frac{1}{q}-\frac{1}{N}$ and we can write

$$
\left(\operatorname{Curl} q_{n} \cdot \nabla\right) u_{n}=\nabla \theta_{n, 2}+\operatorname{Curl} q_{n, 2}
$$

where $\theta_{n, 2}$ is bounded in $L^{q^{*}}(\Omega)$ with $q^{*}=2-\epsilon$ and $q_{n, 2} \in H_{0}^{1}(\Omega)$.

Finally, we can write that

$$
\rho_{n}\left(u_{n} \cdot \nabla\right) u_{n}=\nabla\left(\theta_{n, 1}+\theta_{n, 2}\right)+\operatorname{Curl}\left(q_{n, 1}+q_{n, 2}\right)=\nabla \theta_{n, 3}+\operatorname{Curl} q_{n, 3}
$$

with $\theta_{n, 3}$ bounded in $L^{2-\epsilon}(\Omega)$ and $q_{n, 3} \in H_{0}^{1}(\Omega)$.

These properties verified by $\theta_{n, 3}$ and $q_{n, 3}$ allow us to show that $\rho_{n}$ is bounded in $L^{3}(\Omega)$. Indeed, in (36) we write

$$
\begin{aligned}
{\left[P_{n}\left(\rho_{n}^{2}\right)\right]^{1 / 2} \sum_{i}\left[\int_{\Omega}-\rho_{n} u_{n} \cdot \nabla u_{n} \frac{\nabla p_{i}}{\lambda_{i}} \mathrm{~d} x\right] p_{i} } & =-\left[P_{n}\left(\rho_{n}^{2}\right)\right]^{1 / 2} \sum_{i}\left[\int_{\Omega} \nabla \theta_{n, 3} \frac{\nabla p_{i}}{\lambda_{i}} \mathrm{~d} x\right] p_{i} \\
& =\left[P_{n}\left(\rho_{n}^{2}\right)\right]^{1 / 2} \sum_{i}\left[\int_{\Omega} \theta_{n, 3} p_{i} \mathrm{~d} x\right] p_{i} \\
& =\left[P_{n}\left(\rho_{n}^{2}\right)\right]^{1 / 2} P_{n}\left(\theta_{n, 3}\right)
\end{aligned}
$$


and integrating over $\Omega$, we deduce

$$
\int_{\Omega}\left[P_{n}\left(\rho_{n}^{2}\right)\right]^{1 / 2} P_{n}\left(\theta_{n, 3}\right) \mathrm{d} x \leq\left\|\left[P_{n}\left(\rho_{n}^{2}\right)\right]^{1 / 2}\right\|_{L^{3}(\Omega)}\left\|P_{n}\left(\theta_{n, 3}\right)\right\|_{L^{3 / 2}(\Omega)} .
$$

So, in view of the $L^{2-\epsilon}(\Omega)$ bound on $\theta_{n, 3},(36)$ yields

$$
\left\|P_{n}\left(\rho_{n}^{2}\right)\right\|_{L^{3 / 2}(\Omega)}^{3 / 2} \leq C\left(\left\|P_{n}\left(\rho_{n}^{2}\right)\right\|_{L^{3 / 2}(\Omega)}^{1 / 2}+1\right)
$$

and $P_{n}\left(\rho_{n}^{2}\right)$ is bounded in $L^{3 / 2}(\Omega)$. Thus, $P_{n}\left(\rho_{n}^{2}\right)$ converges weakly to $\ell$ in $L^{3 / 2}(\Omega)$ and we have

$$
\int_{\Omega} P_{n}\left(\rho_{n}^{2}\right) p_{i} \mathrm{~d} x=\int_{\Omega} \rho_{n}^{2} p_{i} \mathrm{~d} x \longrightarrow \int_{\Omega} \ell p_{i} \mathrm{~d} x, \forall p_{i} \in L^{3}(\Omega),
$$

that allows to deduce that $\rho_{n}^{2}$ converges weakly to $\ell$ in $L^{3 / 2}(\Omega)$ and so that $\rho_{n}$ is bounded in $L^{3}(\Omega)$.

Using the same previous arguments, we show that $\rho_{n}$ is bounded in $L^{4}(\Omega)$. Indeed, multiplying (35) by $\left[P_{n}\left(\rho_{n}^{2}\right)\right] p_{i}$ and summing on $i$, we obtain

$$
\begin{array}{r}
{\left[P_{n}\left(\rho_{n}^{2}\right)\right]^{2}=\frac{1}{a}\left[P_{n}\left(\rho_{n}^{2}\right)\right] \sum_{i}\left[\int_{\Omega}\left(-h_{n} u_{n}-\rho_{n} u_{n} \cdot \nabla u_{n}+\rho_{n} f+g_{n}\right) \frac{\nabla p_{i}}{\lambda_{i}} \mathrm{~d} x+\mu \int_{\Omega} \operatorname{div} u_{n} p_{i} \mathrm{~d} x\right] p_{i},} \\
i=1, \ldots, n .
\end{array}
$$

Since $\rho_{n}$ is bounded in $L^{3}(\Omega), \rho_{n}\left(u_{n} \cdot \nabla\right) u_{n}=\nabla \theta_{n, 3}+\operatorname{Curl} q_{n, 3}$ is bounded in $L^{1}(\Omega)^{2}$ and we can find $\theta_{n, 3}$ bounded in $L^{2}(\Omega)$. Using the same computations that in (37), we obtain

$$
\int_{\Omega} P_{n}\left(\rho_{n}^{2}\right) \sum_{i}\left[\int_{\Omega}-\rho_{n} u_{n} \cdot \nabla u_{n} \frac{\nabla p_{i}}{\lambda_{i}} \mathrm{~d} x\right] p_{i} \mathrm{~d} x \leq\left\|P_{n}\left(\rho_{n}^{2}\right)\right\|_{L^{2}(\Omega)}\left\|P_{n}\left(\theta_{n, 3}\right)\right\|_{L^{2}(\Omega)}
$$

and finally, (40) yields

$$
\left\|P_{n}\left(\rho_{n}^{2}\right)\right\|_{L^{2}(\Omega)} \leq C
$$

We conclude that $P_{n}\left(\rho_{n}^{2}\right)$ is bounded in $L^{2}(\Omega)$ and $\rho_{n}$ is bounded in $L^{4}(\Omega)$.

\subsection{Bounds on $\operatorname{div} u_{n}-b \rho_{n}^{2}$ in $W^{1, r}(\Omega)$}

Let us consider once more the equation (23) with $\varphi=\frac{\nabla p_{i}}{\sqrt{\lambda_{i}}}$. Let $P_{n}^{\nabla}$ be the $L^{2}$-projection operator on $\left\{\frac{\nabla p_{1}}{\sqrt{\lambda_{1}}}, \ldots, \frac{\nabla p_{n}}{\sqrt{\lambda_{n}}}\right\}$, we write

$$
P_{n}^{\nabla}\left(\mu \nabla \operatorname{div} u_{n}-a \nabla\left(\rho_{n}^{2}\right)\right)=P_{n}^{\nabla}\left(h_{n} u_{n}+\rho_{n} u_{n} \cdot \nabla u_{n}-\rho_{n} f-g_{n}\right),
$$

so

$$
P_{n}^{\nabla}\left(\nabla\left(\mu \operatorname{div} u_{n}-a \rho_{n}^{2}\right)\right)=P_{n}^{\nabla}\left(h_{n} u_{n}+\rho_{n} u_{n} \cdot \nabla u_{n}-\rho_{n} f-g_{n}\right) .
$$

We show that the right member terms are bounded in $L^{r}(\Omega)^{2}, r<\frac{4}{3}$. Indeed, $\rho_{n} u_{n} \cdot \nabla u_{n}$ is bounded in $L^{r}(\Omega)^{2}$, with $\frac{1}{4}+\frac{1}{p}+\frac{1}{2}=\frac{1}{r}$ and $p<\infty$. In addition, $h_{n} u_{n}$ and $\rho_{n} f$ are bounded in $L^{4 / 3}(\Omega)^{2}$ and $g_{n}$ in $L^{p}(\Omega)^{2}, p<2$. 
Since $P_{n}^{\nabla}\left(\nabla\left(\operatorname{div} u_{n}-b \rho_{n}^{2}\right)\right)=P_{n}^{\nabla}\left(\nabla\left(\pi_{n}\right)\right)$ is bounded in $L^{r}(\Omega)^{2}$, with $b=\frac{a}{\mu}$ then there exists $\pi$ such that $P_{n}^{\nabla}\left(\nabla\left(\pi_{n}\right)\right) \longrightarrow \nabla \pi$ weakly in $L^{r}(\Omega)^{2}$, i.e.

$$
\begin{aligned}
& \int_{\Omega} P_{n}^{\nabla}\left(\nabla \pi_{n}\right) \alpha \mathrm{d} x \longrightarrow \int_{\Omega} \nabla \pi \alpha \mathrm{d} x, \quad \forall \alpha \in L^{r^{*}}(\Omega)^{2}, \frac{1}{r^{*}}=1-\frac{1}{r}, \\
& \int_{\Omega} \sum_{i=1}^{n}\left(\nabla \pi_{n}, \frac{\nabla p_{i}}{\sqrt{\lambda_{i}}}\right) \frac{\nabla p_{i}}{\sqrt{\lambda_{i}}} \alpha \mathrm{d} x \longrightarrow \int_{\Omega} \nabla \pi \alpha \mathrm{d} x, \quad \forall \alpha \in L^{r^{*}}(\Omega)^{2},
\end{aligned}
$$

then choosing $\alpha=\frac{\nabla p_{i}}{\sqrt{\lambda_{i}}} \in L^{r^{*}}(\Omega)^{2}$ and using the orthonormality of the basis, we have

$$
\int_{\Omega} \nabla \pi_{n} \frac{\nabla p_{i}}{\sqrt{\lambda_{i}}} \mathrm{~d} x \longrightarrow \int_{\Omega} \nabla \pi \frac{\nabla p_{i}}{\sqrt{\lambda_{i}}} \mathrm{~d} x
$$

Then, $\nabla \pi_{n}$ is bounded in $L^{r}(\Omega)^{2}$ and $\pi_{n}$ converges strongly to $\pi=\operatorname{div} u-b \overline{\rho^{2}}$ in $L^{r}(\Omega)$ where $\overline{\rho^{2}}$ is the weak limit of $\rho_{n}^{2}$.

\subsection{Compactness Result on $\rho_{n}$}

The crucial bounds on $\rho_{n}$ and $\operatorname{div} u_{n}-b \rho_{n}^{2}$ respectively shown in Sections 1.2 and 1.3 permit to obtain an important compactness result allowing to pass to the limit in the equations. We recall the theorem shown in [6] (Chap. 6, p. 81)

Theorem 1.3. $\rho_{n}$ converges to $\rho$ strongly in $L^{p}(\Omega)$ for all $1 \leq p<4$, when $n$ goes to $+\infty$.

The proof of this result uses in particular the regularization Lemma 2.3 described in [3] and [5] which allows to build up renormalized solutions and uses some convexity properties of these solutions. We just precise the importance of the bound on $\operatorname{div} u_{n}-b \rho_{n}^{2}$ in $W^{1, r}(\Omega)$ which is necessary to prove this result. Indeed, $\operatorname{div} u_{n}-b \rho_{n}^{2}$ is relatively compact in $L^{r}(\Omega)$ and converges strongly in $L^{r}(\Omega)$ to $\operatorname{div} u-b \overline{\rho^{2}}$. This property allows to pass to the limit in the term $\left(\epsilon+\rho_{n}\right)^{\theta}\left[\operatorname{div} u_{n}-b \rho_{n}^{2}\right]$ where $\epsilon>0$ and $0<\theta \leq 1$. Notice that a key for several proofs described in [6] is the convergence of products of this type (see Appendix B of [6]). Indeed, the author shows that the product of $\beta\left(\rho_{n}\right)$ by $\left[\operatorname{div} u_{n}-b \rho_{n}^{\gamma}\right]$ converges weakly to the product of the weak limits of each term i.e. to $\bar{\beta}\left[\operatorname{div} u-b \overline{\rho^{\gamma}}\right]$, for any continuous functions $\beta$ on $[0, \infty)$ such that $\beta(t) t^{(q-\gamma)}$ and $\beta(t) t^{(q / 2)}$ goes to 0 as $t$ goes to $+\infty$.

Finally, this strong convergence result on $\rho_{n}$, given above, allows to pass to the limit in the term $\nabla \rho^{\gamma}$, the real difficulty.

\section{Numerical applications}

We present in this section the numerical results obtained with the compressible and isentropic Navier-Stokes model $(18,19)$ denoted NSCI. In view to obtain a numerical validation, we compare these results with a comparable shallow water model denoted SW, in which the diffusion term depends on $\rho$ and must be evaluated at each time step. Next, we present the numerical results obtained with an analytical case verifying (1, 2) introduced by Weigant [11].

At first, the tests are executed on a simplified studied domain, a square of one unit in length. This particular geometry allows to obtain an analytical expression for the eigenfunctions, solutions of problems $(N)$ and $(D)$ defined in introduction. Next, we present some results executed on a real domain: the dam of Calacuccia in Corsica. In this case, the eigenfunctions are obtained with the finite elements Modulef software. 


\subsection{Comparison between the NSCI model and the SW model}

\subsubsection{The numerical method}

We have shown in Section 1.1 that the following problem, solved with the Galerkin method, had a fixed point and we propose in this section a numerical resolution method of the following equations

$$
\begin{aligned}
& \alpha\left(\rho_{n, m}, \psi\right)+\left(\operatorname{div}\left(\rho_{n, m} v_{n, m}\right), \psi\right)=\left(h_{n, m}, \psi\right), \forall \psi \in H^{1}(\Omega)_{m} \\
& \left(h_{n, m} u_{n, m}, \varphi\right)+\left(\rho_{n, m} v_{n, m} \cdot \nabla v_{n, m}, \varphi\right)+\mu A\left(u_{n, m}, \varphi\right)+a\left(\nabla\left(\rho_{n, m}\right)^{2}, \varphi\right)=\left(\rho_{n, m} f, \varphi\right)+\left(g_{n, m}, \varphi\right), \forall \varphi \in \mathcal{V}_{n}
\end{aligned}
$$

Notice that we set $a=\frac{1}{2}$ in (48), in order to execute the comparison test described in the next section.

We use the global Galerkin method to approximate $u_{n m}$ and $\rho_{n, m}$ which are searched under the form

$$
\begin{aligned}
& u_{n, m}=\sum_{i=1}^{n} a_{i}(t) \varphi_{i}(x)=\sum_{i=1}^{n_{1}} c_{i}(t) U p_{i}(x)+\sum_{i=1}^{n_{2}} d_{i}(t) U q_{i}(x), \\
& \rho_{n, m}=\sum_{l=1}^{m} b_{l}(t) p_{l}(x)
\end{aligned}
$$

where $U p_{i}=\frac{\nabla p_{i}}{\sqrt{\lambda_{i}}}$ and $U q_{i}=\frac{\operatorname{Curl} q_{i}}{\sqrt{\mu_{i}}}$ are solutions of problem $(B)$ presented in introduction.

We describe the numerical resolution method on a time step at time $t$. Replacing $\psi$ in $(47)$ by $p_{k}$, the continuity equation leads to

$$
b_{k}^{j+1}(t)-\Delta t \sum_{i=1}^{n} \sum_{l=1}^{m} a_{i}^{j}(t) b_{l}^{j+1}(t) \int_{\Omega} p_{l} \varphi_{i} \nabla p_{k} \mathrm{~d} x=b_{k}(t-\Delta t),
$$

where $j$ represents the implicit iterations on a time step. Next, the equation (48) leads to a linear system of the form

$$
\begin{aligned}
\frac{1}{\Delta t} \sum_{i=1}^{n} \sum_{l=1}^{m} b_{l}(t-\Delta t) a_{i}^{j+1}(t)\left(\int_{\Omega} p_{l} \varphi_{i} \varphi_{k} \mathrm{~d} x+\mu \Upsilon_{i} \delta_{i k}\right) & =\frac{1}{\Delta t} \sum_{l=1}^{m} \sum_{i=1}^{n} b_{l}(t-\Delta t) a_{i}(t-\Delta t) \int_{\Omega} p_{l} \varphi_{i} \varphi_{k} \mathrm{~d} x \\
+\sum_{l=1}^{m} \sum_{i=1}^{n} \sum_{r=1}^{n} a_{i}^{j}(t) a_{r}^{j}(t) b_{l}^{j+1}(t) & \left(\frac{1}{2} \int_{\Omega} \varphi_{i} \varphi_{r} p_{l} \operatorname{div} \varphi_{k} \mathrm{~d} x+\frac{1}{2} \int_{\Omega} \varphi_{i} \varphi_{r} \nabla p_{l} \varphi_{k} \mathrm{~d} x-\int_{\Omega} p_{l} \operatorname{curl} \varphi_{i} \alpha\left(\varphi_{r}\right) \varphi_{k} \mathrm{~d} x\right) \\
& +\frac{1}{2} \sum_{l=1}^{m} \sum_{s=1}^{m} b_{l}^{j+1}(t) b_{s}^{j+1}(t) \int_{\Omega} p_{l} p_{s} \operatorname{div} \varphi_{k} \mathrm{~d} x+\sum_{l=1}^{m} b_{l}^{j+1}(t) \int_{\Omega} p_{l} f^{n} \varphi_{k} \mathrm{~d} x \quad(52)
\end{aligned}
$$

where $\Upsilon_{i}$ represents the eigenvalues $\lambda_{i}$ or $\mu_{i}$ respectively if $\varphi_{i}$ is equal to $U p_{i}$ or $U q_{i}$.

When we start the method (then $j=0$ ), we set

$$
\begin{aligned}
& b_{l}^{j=0}(t)=b_{l}(t-\Delta t), l=1, \ldots, m, \\
& a_{i}^{j=0}(t)=a_{i}(t-\Delta t), i=1, \ldots, n .
\end{aligned}
$$

Solving (51) and (52), we obtain $b_{l}^{1}$ and $a_{i}^{1}$. Next, we apply a fixed point method for $j \geq 2$ by computing $b_{l}^{j}$ and $a_{i}^{j}$, and we repeat these computations until we get a certain convergence criterion on the sequences, 
where $\epsilon$ is fixed,

$$
\begin{aligned}
& \left\|b_{l}^{j+1}(t)-b_{l}^{j}(t)\right\|_{L^{2}(\Omega)} \leq \epsilon, \\
& \left\|a_{i}^{j+1}(t)-a_{i}^{j}(t)\right\|_{L^{2}(\Omega)^{2}} \leq \epsilon .
\end{aligned}
$$

\subsubsection{Comparison test with a shallow water model}

In order to validate our approximate method, we notice that using (1) we can rewrite (2) as follows

$$
\rho \frac{\partial u}{\partial t}+\rho u \cdot \nabla u-\mu \Delta u+\frac{1}{2} \nabla\left(\rho^{2}\right)=\rho f
$$

and dividing by $\rho$ (noticed that if $\rho_{0}>0$ then $\rho>0$ ), we obtain

$$
\frac{\partial u}{\partial t}+u \cdot \nabla u-\frac{\mu}{\rho} \Delta u+\nabla \rho=f .
$$

As we have analyzed and solved the following shallow water problem [7]

$$
\begin{aligned}
& \frac{\partial \rho}{\partial t}+\operatorname{div}(u \rho)=0, \\
& \frac{\partial u}{\partial t}+u \cdot \nabla u-\nu \Delta u+\nabla \rho=f \\
& u \cdot n=0, \operatorname{curl} u=0 \text { on } \Sigma \\
& u(t=0)=u_{0}(x), \rho(t=0)=\rho_{0}(x), \rho_{0}(x)>0
\end{aligned}
$$

it is natural to compare the solution of $(1,2)$, approximated by $(47,48)$ at each time step, to the solution of the previous shallow water equations in which we set $\nu=\frac{\mu}{\rho(t)}$ where $\rho(t)$ is the solution obtained with $(47,48)$ at time $t$.

\subsubsection{Numerical results}

1. Tests on a square with a constant wind.

Taking a wind $f$ under the form of a gradient, an obvious asymptotic solution of NSCI model, with $u_{0}=0$ and $\rho_{0}=K>0$, is

$$
\bar{u}=0 \quad \text { and the solution of } \quad \frac{1}{2} \nabla \bar{\rho}^{2}=\bar{\rho} f .
$$

So, if $f=(1,0)=\nabla \phi(x)=\nabla(x+C)$ for instance, then

$$
\bar{\rho}=\phi(x)+C^{\prime}
$$

where $C^{\prime}=\frac{1}{\operatorname{meas}(\Omega)} \int_{\Omega} \rho_{0}(x)-\phi(x) \mathrm{d} x$. This example is interesting because we verify that the behaviour of the approximate solution is near the exact solution when $t \longrightarrow \infty$ (notice that we have not proved that $(\bar{\rho}, \bar{u})$ is the asymptotic solution of $(1,2))$. We have represented in Figures 1 and 2 the variation $\rho^{\prime}$ of $\rho$ around its mean value, for both models. We obtain some linear fields, which verify (59).

We represent in Figure 3 the evolution of $E c\left(u_{n}\right)=\frac{1}{2}\left\|u_{n}\right\|_{L^{2}(\Omega)^{2}}^{2}=\frac{1}{2} \sum_{i=1}^{n} a_{i}(t)^{2}$ for both models (which corresponds to kinetic energy if $\rho$ is constant but we employ this term by abuse). This figure shows that the velocity initialized to zero, oscillates and goes to zero when $t$ goes to $\infty$. 


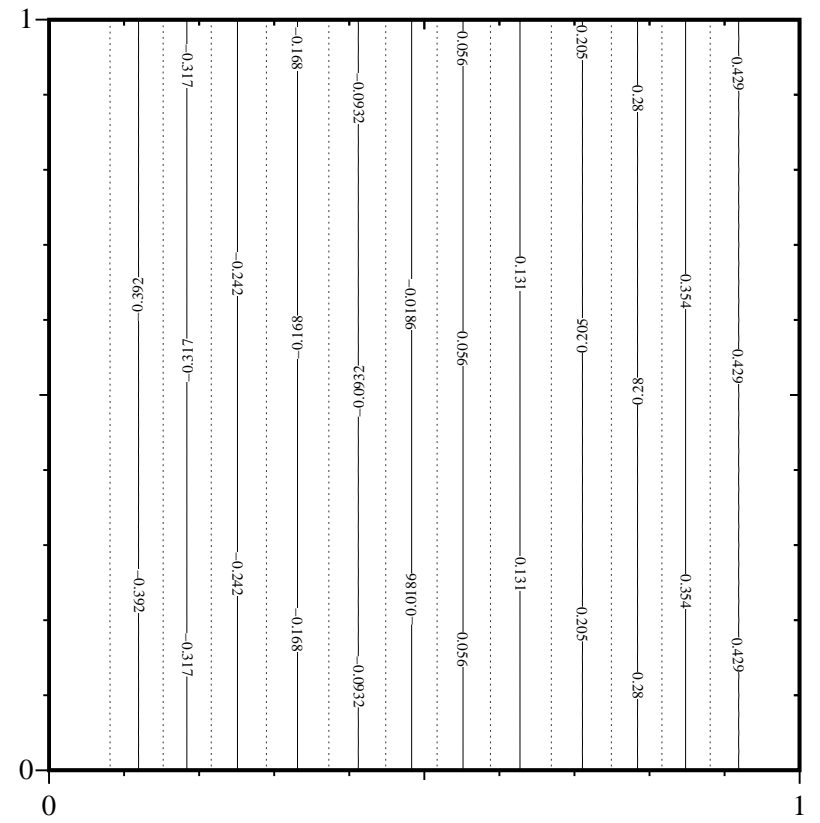

FiguRE 1. Variation of $\rho$ for NSCI model.

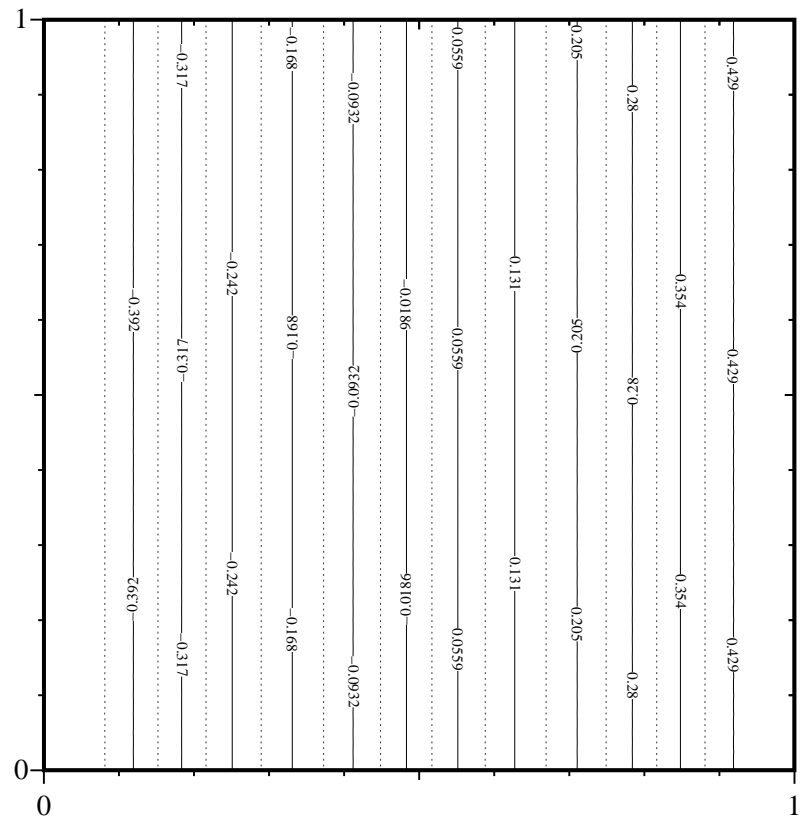

Figure 2. Variation of $\rho$ for SW model.

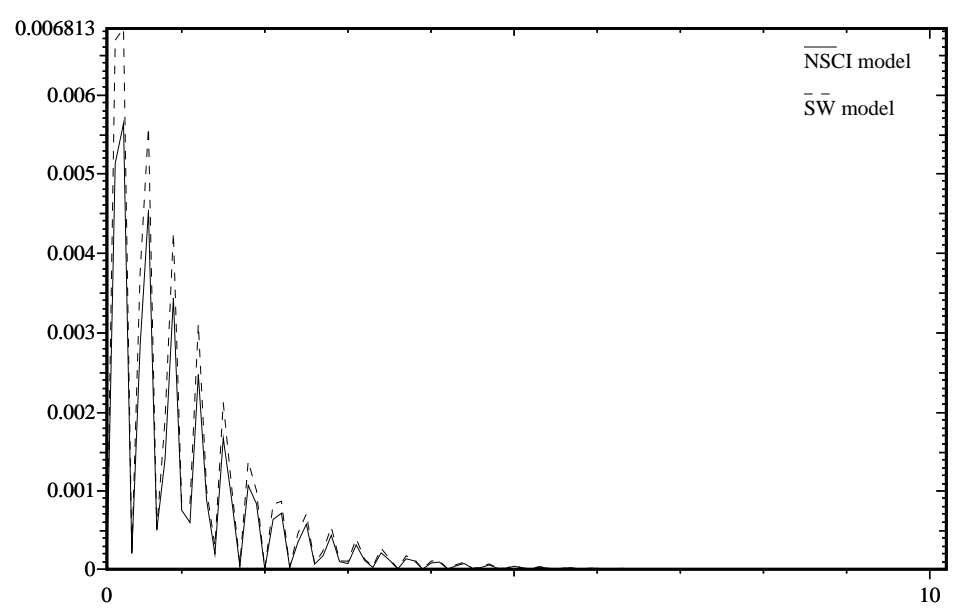

Figure 3. Kinetic energy.

Next, we have represented in Figure 4 the evolution of $\|\nabla \rho-f\|_{L^{2}(\Omega)^{2}}$ for both models (notice that the curves are quasi-identical) in order to verify that this difference goes to zero.

2. Tests on a "real" domain: the dam of Calacuccia (Corsica).

The Figure 5 represents the bathymetry of the dam of Calacuccia whose the maximal depth is approximately fifty meters. The tests are computed with a west dominant wind characteristic of this geographical area (Fig. 6). The results obtained for both models are quasi identical and for the velocity fields, we observe a circulation in the direction of the wind close to the coasts (where the height of water is low) and 


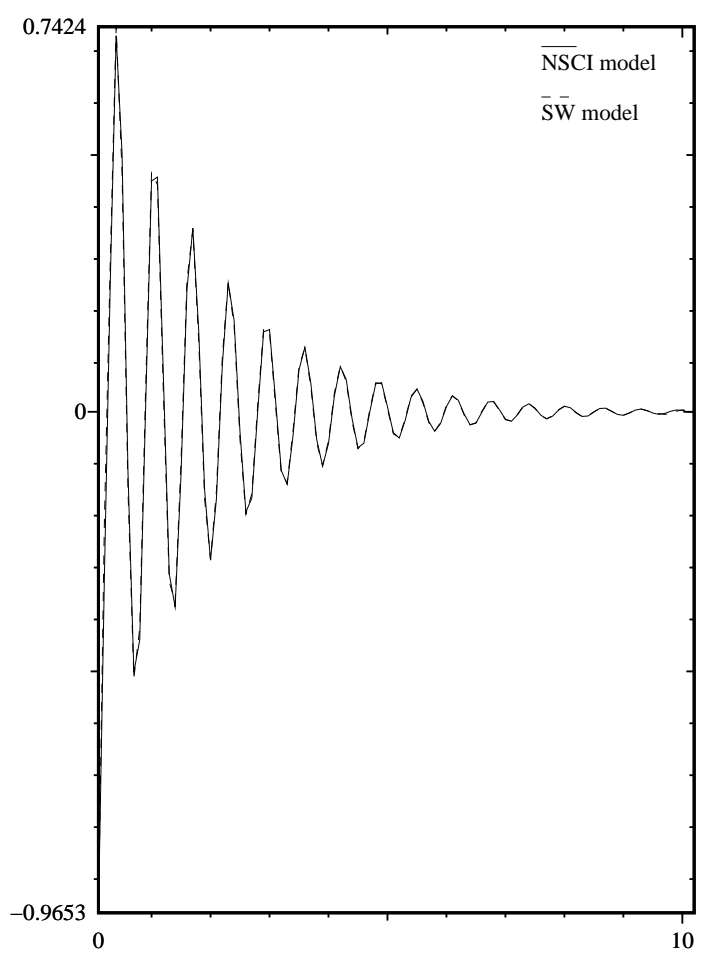

Figure 4. Evolution of $\|\nabla \rho-f\|_{L^{2}(\Omega)^{2}}$.

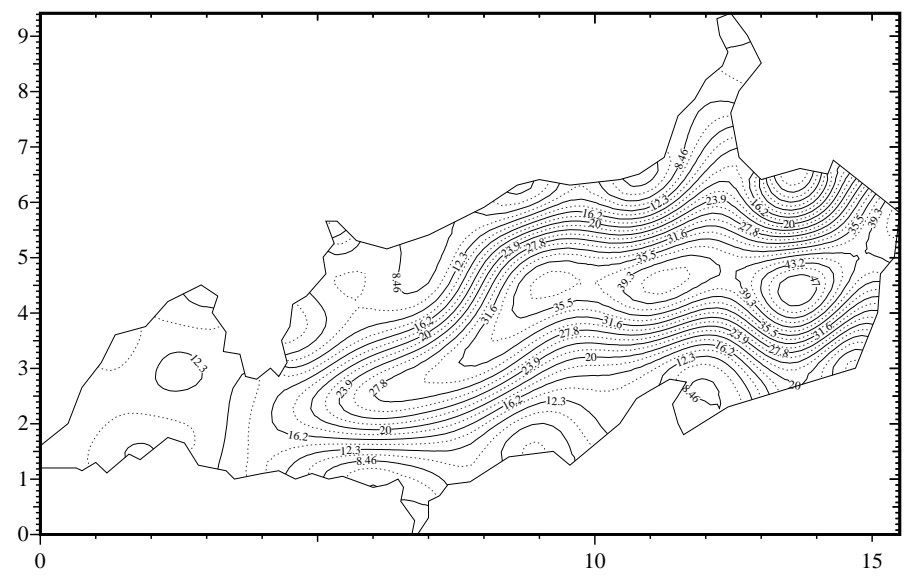

Figure 5. Smoothed bathymetry.

a recirculation by the center of the domain where the depth is most important (Figs. 7 and 8). The difference observed on the maximal velocity is probably due to the truncating level of the Galerkin method. Indeed, for these computations, we have used the 30 first eigenvectors for each $(N)$ and $(D)$ problem and on a complex domain, contrary to the square case, we need a higher number of modes for better representing the solution. More precision is more expensive and this is mainly due to the nonlinear terms. We remark in the Figures 9 and 10 that the surface elevation is only influenced by the wind. 


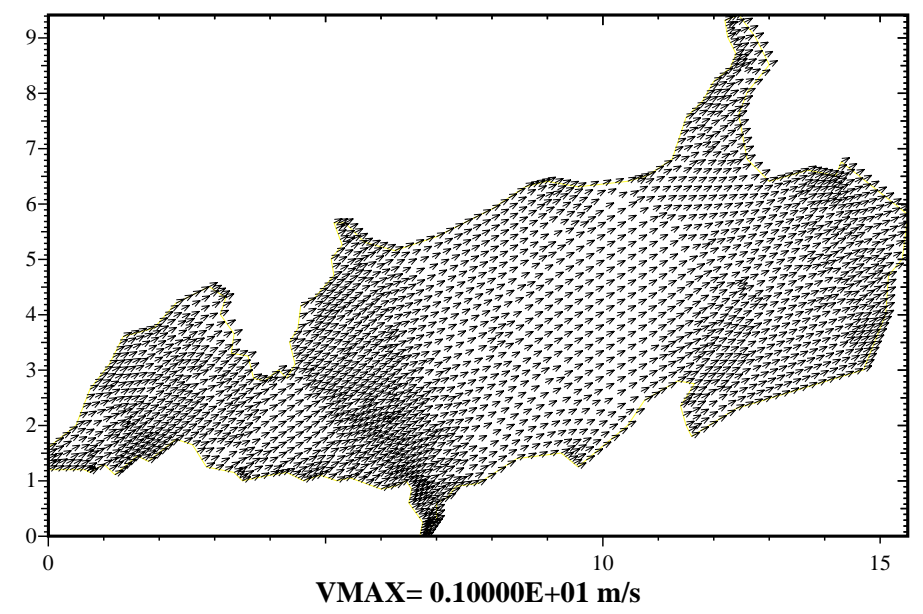

FIGURE 6 . Wind.

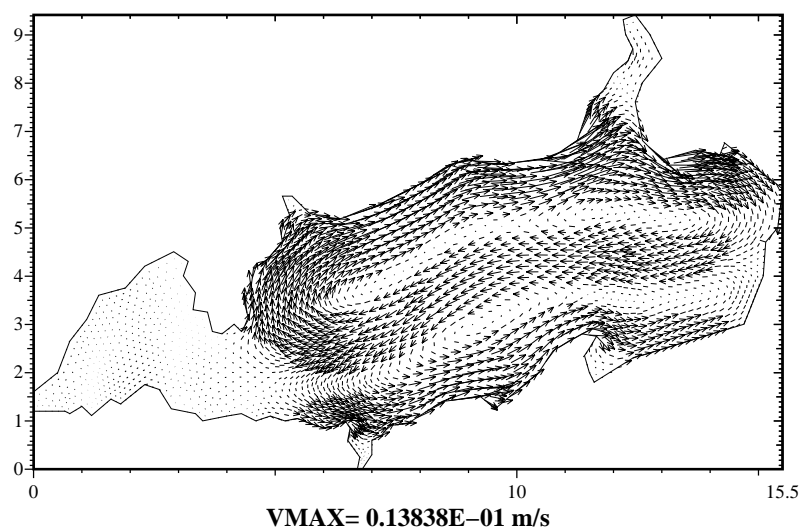

FiguRe 7. $u$ for NSCI model.

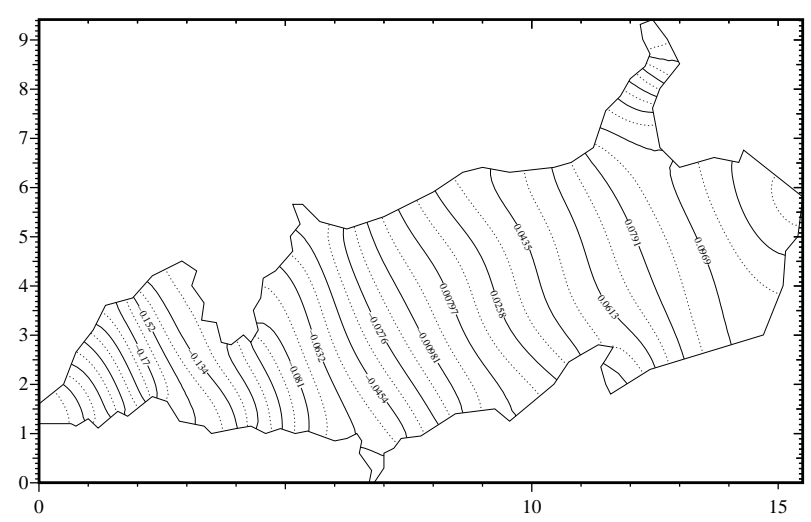

FiguRe 9. Variation of $\rho$ for NSCI model.

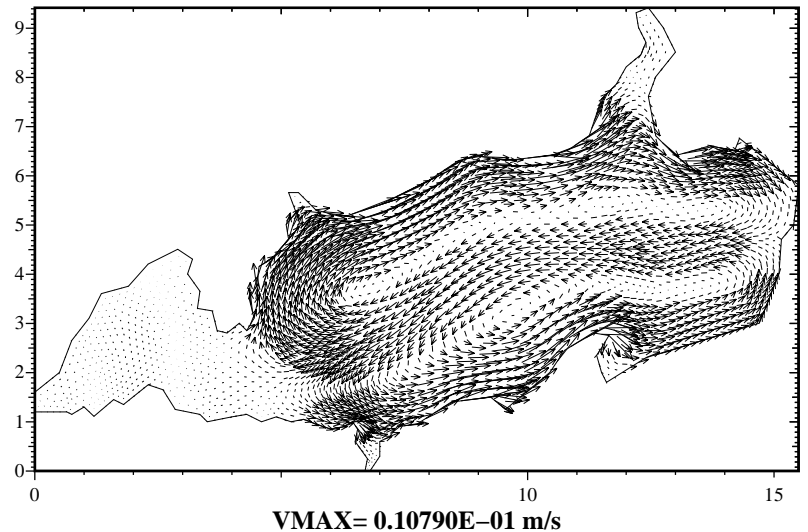

Figure 8. $u$ for SW model.

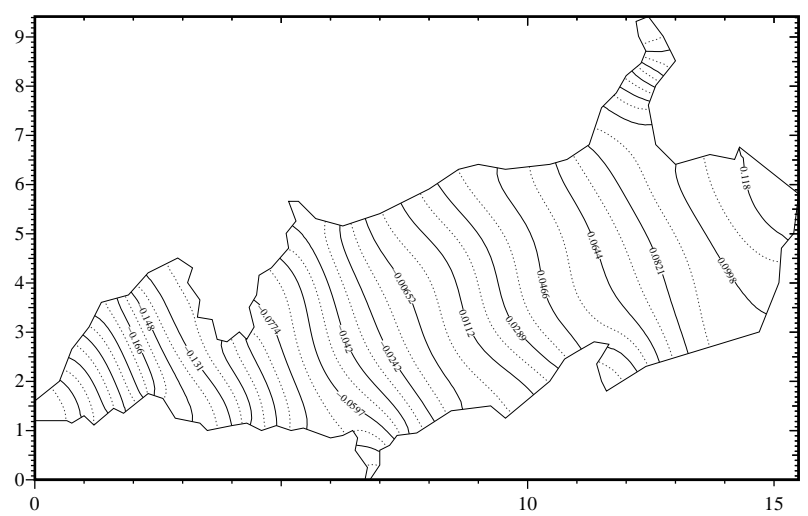

Figure 10. Variation of $\rho$ for SW model. 


\subsection{An analytical test}

\subsubsection{An example by Weigant [11]}

We numerically implement an analytical case presented by Weigant. This example indicates the existence of smooth solutions $(\rho, u)$ of $(1,2)$ which blow up in finite time, namely the maximal existence interval of the solution is finite. Weigant uses a result proved in [10] which corresponds to a local existence result for a small time that we recall here.

Let $f \in L^{q}(\Omega \times(0, T)), q>N, u_{0} \in W^{2-\frac{2}{q}, q}(\Omega), \nabla \rho_{0} \in L^{q}(\Omega), 0<m \leq \rho_{0}(x) \leq M<\infty$ then there exists $T_{0} \in(0, T]$ such that for each $0<t_{0} \leq T_{0}$ in $Q_{t_{0}}=\Omega \times\left(0, t_{0}\right)$ a generalized solution of the problem exists and is unique. This solution verifies $u \in W_{x, t}^{2,1, q}\left(Q_{t_{0}}\right)$ i.e. $u \in L^{q}\left(0, t_{0} ; W^{2, q}(\Omega)\right), \frac{\partial u}{\partial t} \in L^{q}\left(\Omega \times\left(0, t_{0}\right)\right)$ and $\rho \in L^{\infty}\left(0, t_{0} ; W^{1, q}(\Omega)\right), \frac{\partial \rho}{\partial t} \in L^{\infty}\left(0, t_{0} ; L^{q}(\Omega)\right)$ with $0<m_{1} \leq \rho(x, t) \leq M_{1}<\infty$.

So, from this result, Weigant writes his assertion.

Proposition 2.1. If $0 \leq \gamma<1+\frac{1}{N-1}$, then there exists functions $u_{0}, \rho_{0}, f$ satisfying the conditions of [10] for which locally in time a generalized solution of (1, 2) with Dirichlet boundary conditions cannot be extended to $Q_{T}$.

In order to illustrate this result, he constructs the following analytical case

$$
\begin{aligned}
& Q=B \times(0,1), \quad \bar{Q}=B \times[0,1], \quad r=\left(x_{1}^{2}+x_{2}^{2}\right)^{1 / 2}, \quad 0 \leq t \leq 1, \\
& B=\left\{x=\left(x_{1}, x_{2}\right) \in \mathbb{R}^{2} / r \leq 1\right\}, \\
& u_{j}(x, t)=\frac{1}{r} x_{j} u(r, t), \quad u_{0 j}(x)=\frac{1}{r} x_{j} u_{0}(r), \quad f_{j}(x, t)=\frac{1}{r} x_{j} f(r, t), \\
& \rho(x, t)=\rho(r, t), \quad \rho_{0}(x)=\rho_{0}(r), \quad 1 \leq j \leq 2,
\end{aligned}
$$

where the analytical solution $(u(r, t), \rho(r, t), f(r, t))$ is the following

$$
\begin{aligned}
& u(r, t)=-\frac{2 \alpha r s(1-t)^{2 s-1}\left(1-r^{2 l}\right)}{\left[1+(1-t)^{2 s}\right]\left[(N-2 \alpha l) r^{2 l}+N(1-t)^{2 s}\right]} \\
& \rho(r, t)=\frac{\left[1+(1-t)^{2 s}\right]^{\alpha}\left[(N-2 \alpha l) r^{2 l}+N(1-t)^{2 s}\right]}{\left[r^{2 l}+(1-t)^{2 s}\right]^{\alpha+1}} \\
& f(r, t)=u_{t}+u u_{r}+\frac{1}{\rho} P_{r}-\frac{\mu}{\rho}\left(\frac{1}{r^{N-1}}\left(r^{N-1} u\right)_{r}\right)_{r} \\
& u_{0}(r)=u(r, 0), \quad \rho_{0}(r)=\rho(r, 0),
\end{aligned}
$$

with $P=a \rho^{\gamma} . l, s$ are natural numbers and $\alpha$ satisfies the inequality $0<2 \alpha l<N$. This example is constructed such that $\rho(r, t) \longrightarrow+\infty$ when $t \longrightarrow 1$ and $r \longrightarrow 0$.

For the numerical application, in order to respect the previous restrictions, we set $\alpha=\frac{1}{2}, s=l=1, a=\frac{2}{3}$ and $\gamma=\frac{3}{2}$.

Notice that Weigant considers some Dirichlet boundary conditions but since, the analytical velocity $u(r, t)$ verifies the condition $\operatorname{curl} u=0$ on the boundary, the boundary conditions that we consider in this paper are satisfied.

We consider a system of coordinates $(r, \theta)$ and since the two-dimensional velocity $u(x, t)$ is such that $u \cdot e_{\theta}=0$, the problem can be reduced to a one dimensional problem and we compute only $u \cdot e_{r}$. 


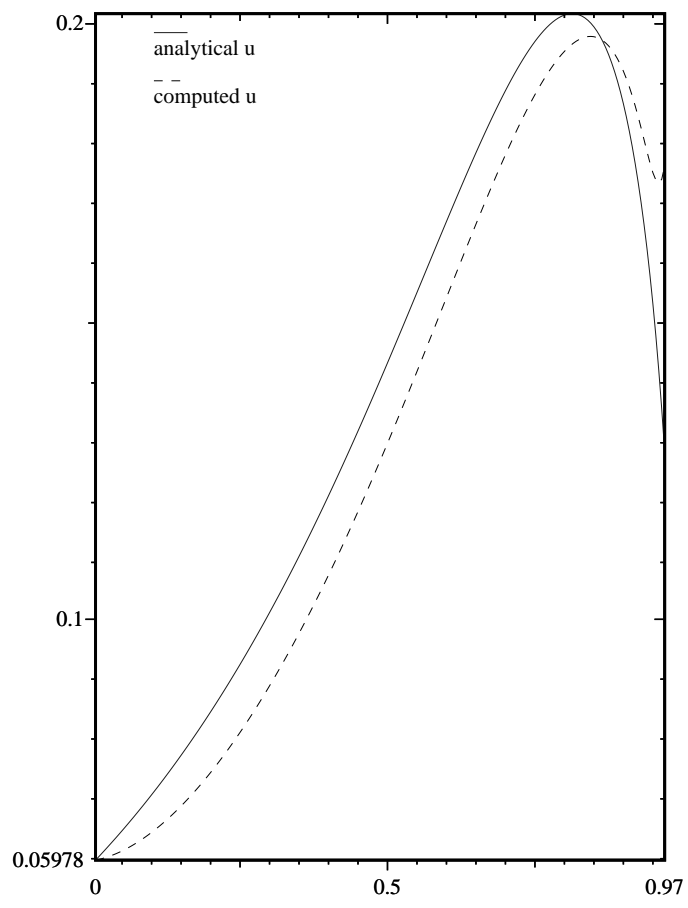

FiguRE 11. $L^{2}(\Omega)^{2}$ norms of velocities $\left(\Delta t=5 \times 10^{-3}\right)$.

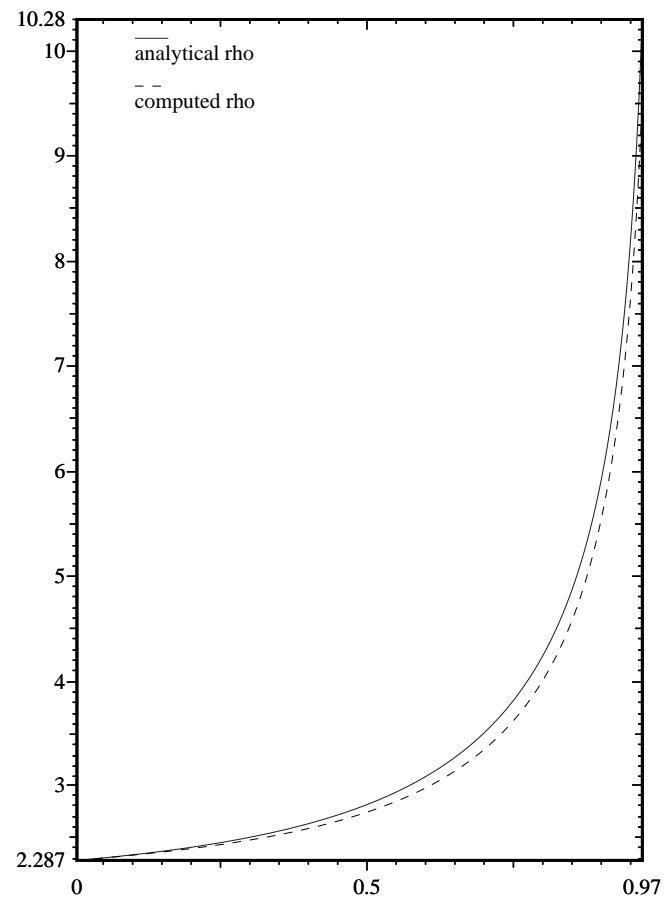

FiguRE 12. $L^{2}(\Omega)$ norms of densities $\left(\Delta t=5 \times 10^{-3}\right)$.

The time-discretized equations in coordinates $(r, \theta)$ are

$$
\begin{aligned}
& \alpha \rho+\frac{\partial}{\partial r}(\rho u)+\frac{\rho u}{r}=h, \\
& h u+\rho u \frac{\partial u}{\partial r}-\mu\left(\frac{1}{r} \frac{\partial}{\partial r}\left(r \frac{\partial u}{\partial r}\right)-\frac{u}{r^{2}}\right)+\frac{2}{3} \frac{\partial \rho^{3 / 2}}{\partial r}=\rho f_{\text {ana }}+g,
\end{aligned}
$$

where $f_{\text {ana }}$ is given by $(66)$.

The numerical method is also solved with the global Galerkin method. In one-dimensional case, the basis functions are sine or cosine functions. Then, the unknowns are searched under the form

$$
\begin{aligned}
& \rho_{n}=b_{0}(t)+\sum_{j=1}^{n} b_{j}(t) \sqrt{2} \cos (j \pi x) \\
& u_{n}=\sum_{i=1}^{n} a_{i}(t) \sqrt{2} \sin (i \pi x)
\end{aligned}
$$

where the $a_{j}(t)$ and $b_{j}(t)$ are coefficients that we have to found.

The tests that we have executed show us that $\rho$ blows up when $t \longrightarrow 1$ (Fig. 12). We represent in Figures 11 and 12 the comparison between the analytical and computed velocities or densities. We respectively represent in Figures 13 and 14 the fields of analytical $\rho$ and computed $\rho$ when $t \longrightarrow 1$ (more precisely when the solution blows up at $t=0.97)$ in order to verify that when $t \longrightarrow 1$, then $\rho \longrightarrow+\infty$ when $r \longrightarrow 0(r \in[0,1]$ is represented on $\mathrm{X}$-axis). 


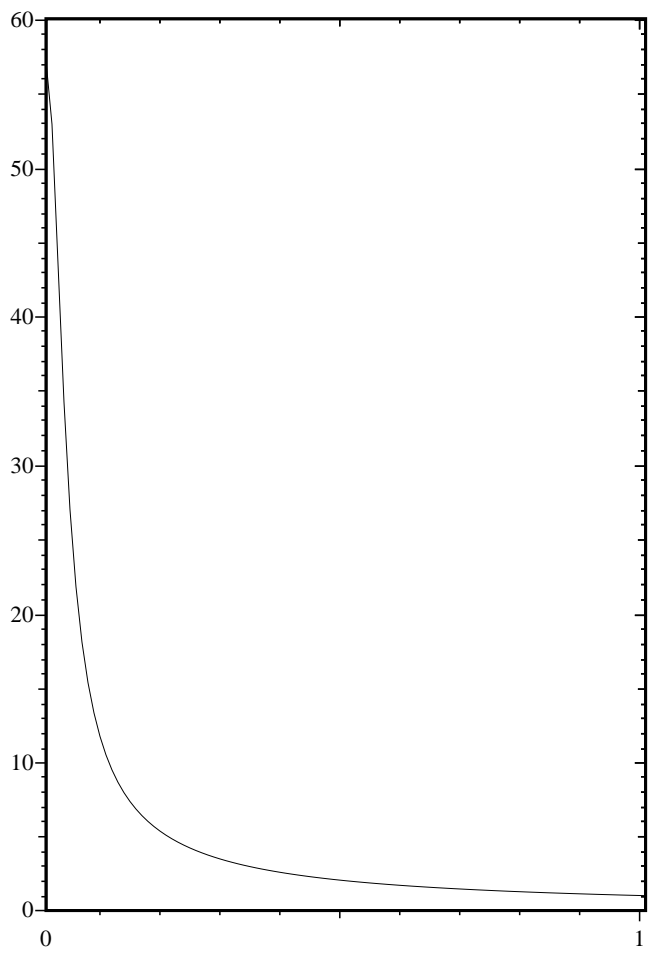

Figure 13. Analytical $\rho$ for $t=0.97$.

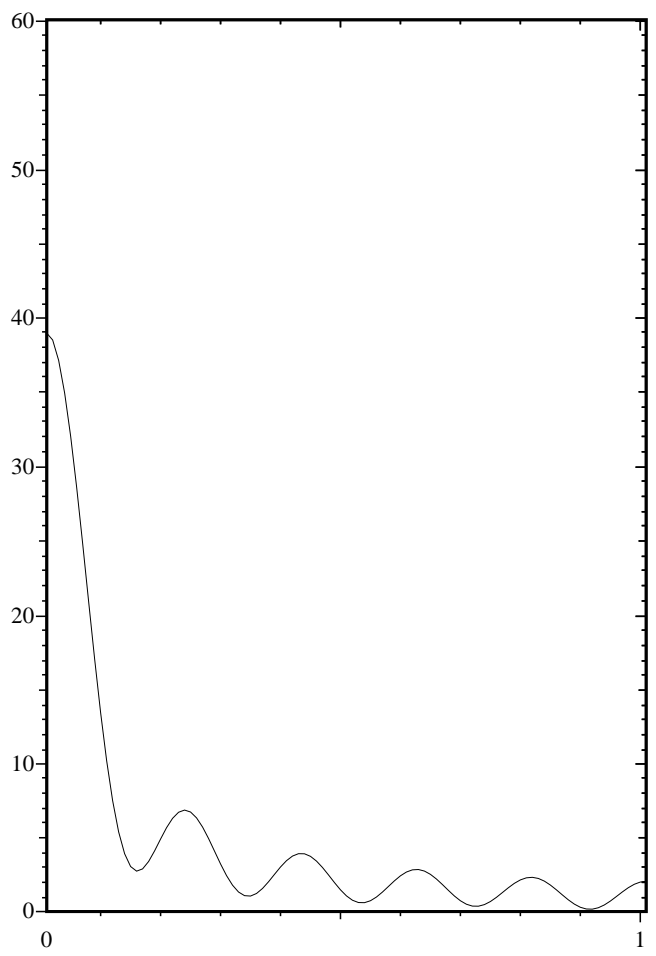

Figure 14. Computed $\rho$ for $t=0.97$.

\subsubsection{Regularizing role of penalization term}

To regularize the solution, we add a penalization term $\epsilon \Delta \rho, \epsilon>0$ in continuity equation and we obtain the following problem

$$
\begin{aligned}
& \alpha \rho_{n}+\frac{\partial \rho_{n} v_{n}}{\partial r}+\frac{\rho_{n} v_{n}}{r}-\frac{\epsilon}{r} \frac{\partial}{\partial r}\left(r \frac{\partial \rho_{n}}{\partial r}\right)=h_{n}, \\
& h_{n} u_{n}+\rho_{n} v_{n} \frac{\partial v_{n}}{\partial r}+\frac{\epsilon}{2 r} \frac{\partial}{\partial r}\left(r \frac{\partial \rho_{n}}{\partial r}\right) v_{n}-\mu\left(\frac{1}{r} \frac{\partial}{\partial r}\left(r \frac{\partial u_{n}}{\partial r}\right)+\frac{u_{n}}{r^{2}}\right)+\frac{2}{3} \frac{\partial \rho_{n}^{3 / 2}}{\partial r}=\rho_{n} f_{\text {ana }}+g_{n} .
\end{aligned}
$$

We can see in Figure 16 that the penalization term avoids the explosion of $\rho$ (which is in agreement with the theory [6]). Indeed, the computed $\rho$ is attenuated about $t=1$ and does not blow up. There is a good adequacy between the computed and the analytical velocities (Fig. 15).

Next, we have studied the size order of penalization term in the penalized continuity equation. Multiplying equation (72) by $\rho$ and integrating over $[0,1]$, we obtain

$$
\alpha \int_{0}^{1} \rho_{n}^{2} \mathrm{~d} r+\int_{0}^{1}\left(\frac{\partial \rho_{n} v_{n}}{\partial r}+\frac{\rho_{n} v_{n}}{r}\right) \rho_{n} \mathrm{~d} r-\epsilon \int_{0}^{1} \frac{1}{r} \frac{\partial}{\partial r}\left(r \frac{\partial \rho_{n}}{\partial r}\right) \rho_{n} \mathrm{~d} r=\int_{0}^{1} h_{n} \rho_{n} \mathrm{~d} r .
$$

We represent in Figure 17 the evolution of the term $-\epsilon \int_{0}^{1} \frac{1}{r} \frac{\partial}{\partial r}\left(r \frac{\partial \rho_{n}}{\partial r}\right) \rho_{n} \mathrm{~d} r$. This curve presents a pick for $t=1$ and is almost zero elsewhere. We can see that the penalization term rectifies the solution where there is an explosion. This fact is represented in Figure 18 on which the curve 1 in continuous line represents the term $\int_{0}^{1}\left(\alpha \rho_{n}^{2}-h_{n} \rho_{n}\right) \mathrm{d} r$ and the curve 2 the term $\int_{0}^{1}\left(\alpha \rho_{n}^{2}-h_{n} \rho_{n}\right) \mathrm{d} r-\epsilon \int_{0}^{1} \frac{1}{r} \frac{\partial}{\partial r}\left(r \frac{\partial \rho_{n}}{\partial r}\right) \rho_{n} \mathrm{~d} r$. 


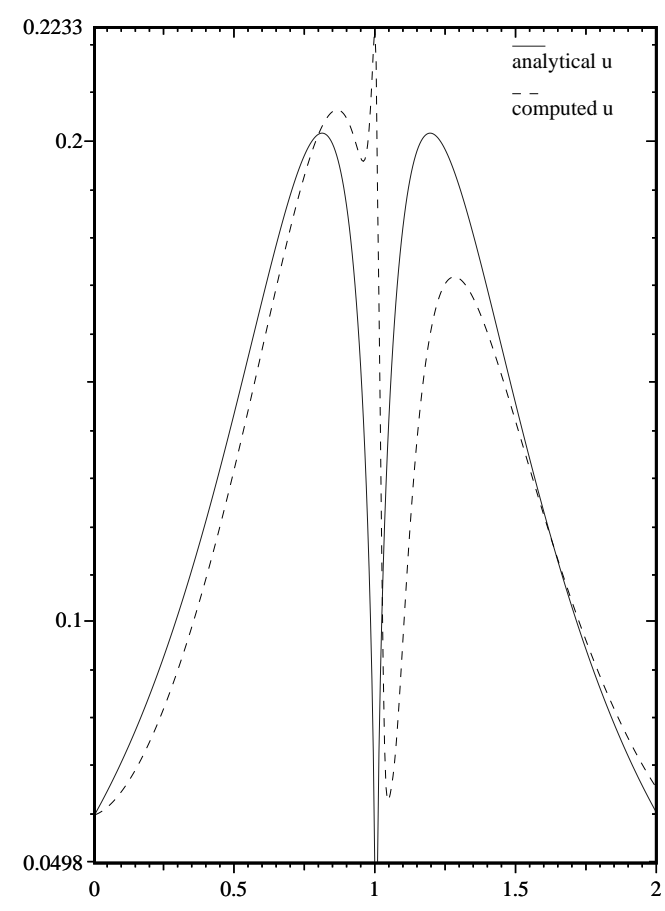

FiguRE 15. $L^{2}(\Omega)^{2}$ norms of velocities $\left(\epsilon=10^{-16}\right)$.

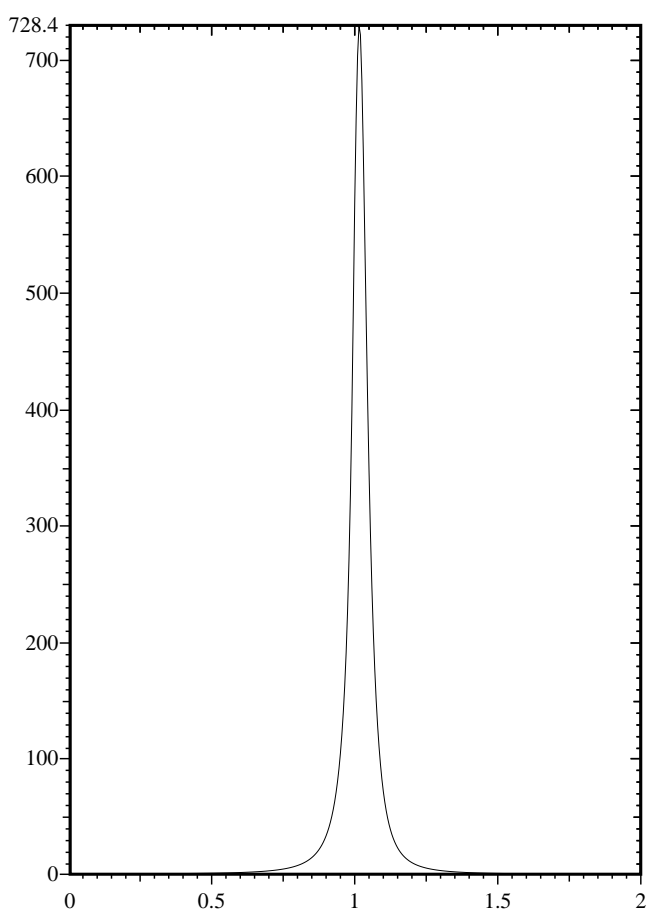

FiguRE 17. Representation of term $-\epsilon \int_{0}^{1} \frac{1}{r} \frac{\partial}{\partial r}\left(r \frac{\partial \rho_{n}}{\partial r}\right) \rho_{n} \mathrm{~d} r$.

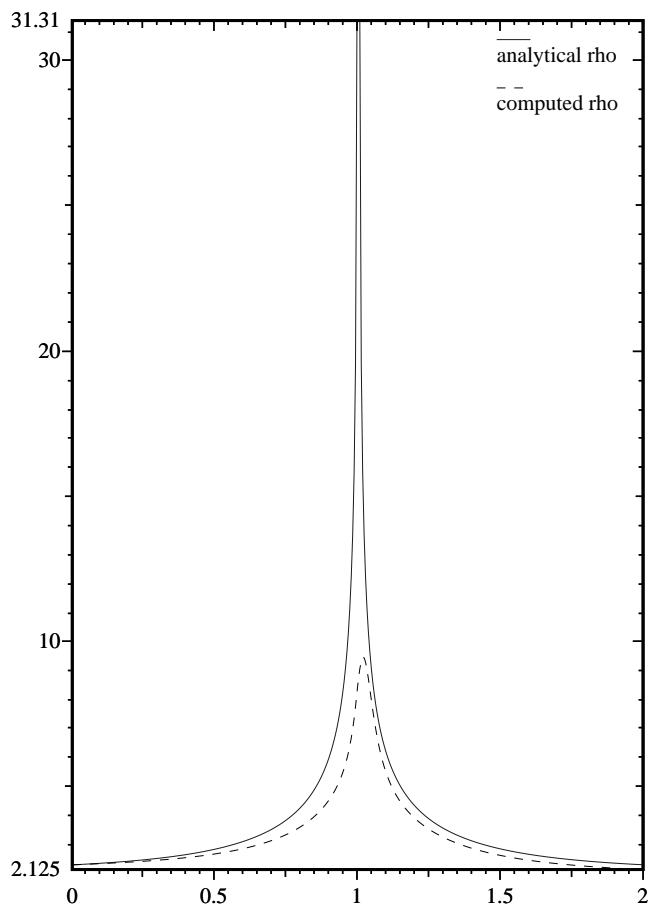

FiguRE $16 . L^{2}(\Omega)$ norms of $\rho\left(\epsilon=10^{-16}\right)$.

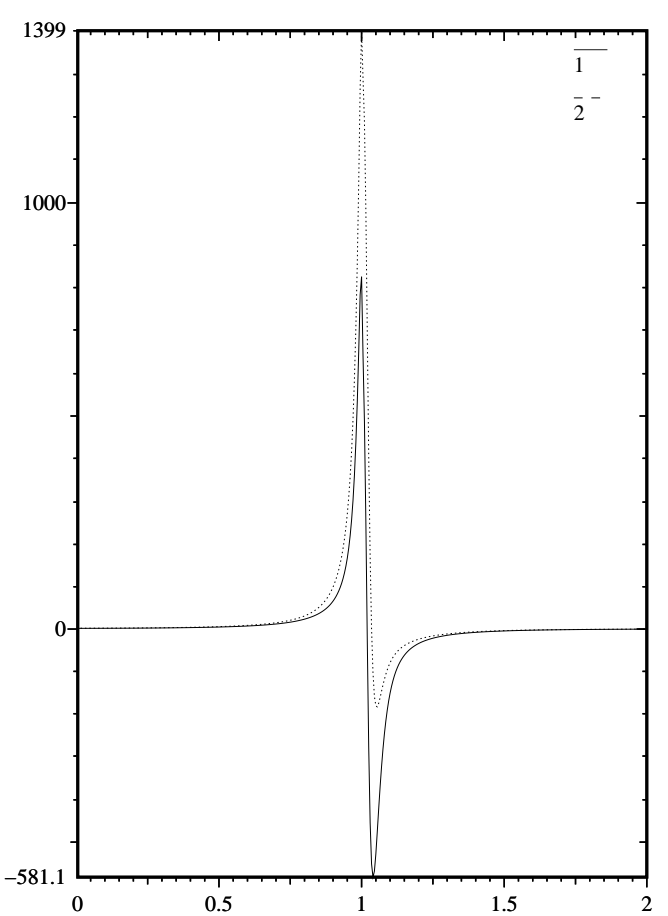

Figure 18. Role of penalization term. 


\section{REFERENCES}

[1] R. Coifman, P.L. Lions, Y. Meyer and S. Semmes, Compensated-compactness and Hardy spaces. J. Math. Pures Appl. 72 (1993) 247-286.

[2] R.J. DiPerna and P.L. Lions, On the cauchy problem for boltzman equations: global existence and weak stability. C.R. Acad. Sci. Paris Sér. I Math. 306 (1988) 343-346.

[3] R.J. DiPerna and P.L. Lions, Ordinary differential equations, transport theory and sobolev spaces. Invent. Math. 98 (1989) $511-547$.

[4] V. Girault and P.A. Raviart, Finite Elements Methods of the Navier-Stokes Equations. Springer-Verlag (1986).

[5] P.L. Lions, Mathematical Topics in Fluid Mechanics, Incompressible models. Vol. 1, Oxford Science Publications (1996)

[6] P.L. Lions, Mathematical Topics in Fluid Mechanics, Compressible models. Vol. 2, Oxford Science Publications (1998).

[7] B. Di Martino, F.J. Chatelon and P. Orenga, The nonlinear Galerkin's method applied to the shallow water equations. Math. Models Methods Appl. Sci. 9 (1999) 825-854.

[8] P. Orenga, Analyse de quelques problèmes d'océanographie physique. Ph.D. thesis, Université de Corse, Corte (1992).

[9] P. Orenga, Construction d'une base spéciale pour la résolution de quelques problèmes non linéaires d'océanographie physique en dimension deux, in Nonlinear partial differential equations and their applications, D. Cioranescu and J.L. Lions, Vol. 13. Longman, Pitman Res. Notes Math. Ser. 391 (1998) 234-258.

[10] V.A. Solonnikov, Zap. Nauchn. Sem. Leningrad. Otdel. Mat. Inst. Steklov. (LOMI) 56 (1976) 128-142. English translation in J. Soviet Math. 14 (1980) 1120-1133.

[11] V.A. Weigant, An exemple of non-existence globally in time of a solution of the Navier-Stokes equations for a compressible viscous barotropic fluid. Russian Acad. Sci. Doklady Mathematics 50 (1995) 397-399.

[12] E. Zeidler, Fixed-point theorems, in Nonlinear Functional Analysis and its Applications, Vol. 1, Springer-Verlag (1986).

To access this journal online:

www.edpsciences.org 